\title{
The efficacy of hyperbaric oxygen in hemorrhagic stroke: experimental and clinical implications
}

Robert P. Ostrowski, Katarzyna Stępień, Emanuela Pucko, Ewa Matyja

Department of Experimental and Clinical Neuropathology, Mossakowski Medical Research Centre, Polish Academy of Sciences, Warsaw, Poland

Submitted: 30 June 2016

Accepted: 10 September 2016

Arch Med Sci 2017; 13, 5: 1217-1223

DOI: 10.5114/aoms.2017.65081

Copyright $\odot 2017$ Termedia \& Banach

\section{Abstract}

Hemorrhagic stroke, accounting for $10-30 \%$ of stroke cases, carries high rates of morbidity and mortality. This review presents the current knowledge on the efficacy of hyperbaric oxygen (HBO)-based modalities in the preclinical research on hemorrhagic stroke. Both preconditioning and post-treatment with $\mathrm{HBO}$ are considered as prospective therapeutic options. High efficacy of HBO therapy (HBOT) for brain hemorrhage has been noted. We found that moderate hyperbaric pressures appear optimal for therapeutic effect, while the therapeutic window of opportunity is short. HBO preconditioning offers more modest neuroprotective benefit as compared to HBO post-treatment for experimental intracerebral hemorrhage. We advocate for mandatory calculations of percent changes in the experimentally investigated indexes of $\mathrm{HBO}$ effectiveness and stress the need to design new clinical trials on $\mathrm{HBO}$ for hemorrhagic stroke.

Key words: intracerebral hemorrhage, subarachnoid hemorrhage, brain edema, preclinical research.

\section{Introduction}

Hyperbaric oxygen (HBO, 100\% oxygen above 1.0 atm absolute; ATA) may not seem to be an intuitive intervention for intracerebral hemorrhage $(\mathrm{ICH})$, as opposed to cerebral ischemia where it may augment oxygen supply to the ischemic territory [1]. It is more difficult to conceptualize the benefit from HBO therapy (HBOT) for bleeding in the brain. However, this type of stroke also involves a hypoxic component in the tissue surrounding the hemorrhagic lesion, with cells striving for oxygen [2]. Venules and arterioles contract in response to HBOT, which may theoretically attenuate the bleeding. It also may lead to a diminution in cerebral blood volume and edema, providing relief from increased intracranial pressure and thus an improved microcirculation [3]. The HBO may also target the phenomena underlying secondary brain injury after hemorrhage, including oxidative stress, apoptosis, autophagy, and inflammation, while boosting brain regeneration and functional performance [4].

Worldwide, across distinct human populations, from $10 \%$ to $27 \%$ of strokes are hemorrhagic [5]. In the category of hemorrhagic strokes is the subarachnoid hemorrhage (SAH), in which the blood, most often from a ruptured aneurysm of an intracranial artery, extravasates between the arachnoid and pia mater membranes [6]. The ICH refers to bleeding with-

\author{
Corresponding author: \\ Prof. Ewa Matyja MD, PhD \\ Mossakowski Medical \\ Research Centre \\ Polish Academy \\ of Sciences \\ Warsaw, Poland \\ 5 Pawinskiego St \\ 02-106 Warsaw, Poland \\ Phone: +48 226086543 \\ E-mail: ematyja@imdik.pan.pl
}


in the tissue of the brain, most often the basal ganglia [7].

Animal models of hemorrhagic stroke are useful for assessing cerebral pathophysiological phenomena modified by investigational therapies as well as underlying molecular alterations [8]. These models also allow for determination of the effectiveness of $\mathrm{HBO}$ with respect to diverse and clinically relevant hemorrhagic brain injuries and treatment regimens, including both post-treatment and preconditioning approaches. Assessing HBO effectiveness requires clearly defined indexes of hemorrhagic injuries. Targeting brain edema, which is a good indicator of hemorrhage-induced brain injury, may provide a focus for therapeutic interventions. As for cerebral ischemia, infarct volume or the extent of cell damage in vulnerable anatomical brain regions is often evaluated. For hemorrhages, on the other hand, investigators must select the analyzed areas of cell damage, which can be subjective. In addition, lesion volume is not commonly determined in hemorrhagic stroke models. Instead, percent reductions in water content referenced to levels in the control tissue may provide an estimate of $\mathrm{HBO}$ effectiveness.

Preclinical studies have shown that edema severity strongly correlates with neurologic impairments after ICH [9]. Brain edema reduction also allows for an approximate comparison of $\mathrm{HBO}$ effectiveness between hemorrhagic and ischemic brain injuries. The purpose of this review was to identify hemorrhagic conditions and treatment regimens that are associated with the highest percent reductions in brain injury indicators with HBO therapy, with the goal of facilitating its transition to clinical trials.

\section{Subarachnoid hemorrhage and hyperbaric oxygen}

The SAH accounts for $5-10 \%$ of all strokes [10], affects relatively young people, carries high mortality, and leaves one third of patients with a lasting disability [11]. Upon the initial bleed, there is a profound decrease in cerebral blood flow, which could be considered as a mechanism for reducing bleeding; however, if the hypoperfusion persists beyond several minutes, brain ischemia arises and cell hypoxia ensues [12]. This phenomenon may explain the presence of injured cells remotely from extravasated blood collections. Especially because of this distant ischemic injury, HBO can be considered as a potentially beneficial therapeutic option.

Until recently, $\mathrm{HBO}$ was not used in the early period after $\mathrm{SAH}$, and its clinical utility for SAH was limited to patients who developed cerebral vasospasm following the subarachnoid bleeding [13]. The SAH pathophysiology includes global cerebral ischemia arising by a mechanism of an immediate increase in the intracranial pressure and a drop in cerebral perfusion pressure after bleeding, which induces robust expression of hypoxia-inducible factor- $1 \alpha$ that peaks at $24 \mathrm{~h}$ after the ictus [14, 15].

The robustness of endpoint measures for SAH is limited because morphological injury after SAH is not clearly delineated. Therefore, in the assessment of HBO efficacy after hemorrhage, researchers have used cell counts and indexes of brain injury that relate to brain edema and swelling in predefined regions of interest. In one study [15], $\mathrm{HBO}$ administered at 2.8 ATA for $2 \mathrm{~h}$ starting at 60 min after an experimental SAH reduced the increase in wet brain weight by $56.3 \%$ and reduced the increase in blood volume by $61.45 \%$. The use of $\mathrm{HBO}$ was also associated with a statistically significant improvement in neurological function in that study. In another investigation, the action of $\mathrm{HBO}$ at the above parameters led to a decrease in malondialdehyde concentration in the cerebral cortex by $64.6 \%$ at $6 \mathrm{~h}$ and by $81.75 \%$ at $24 \mathrm{~h}$ after bleeding, as compared to the untreated group. That study confirmed that HBO can reduce oxidative stress-induced brain damage after SAH [16].

In a single hemorrhage SAH model producing $50 \%$ or more angiographic vasospasm, HBO at 3 ATA for $1 \mathrm{~h}$, applied one day after SAH, reduced the increment in neurological severity score by $62.5 \%$ at 3 days after $\mathrm{SAH}$, and by $72.6 \%$ at 10 days after $\mathrm{SAH}$, compared to the results with a normobaric oxygen treatment [17]. However, not all indicators of SAH-induced brain injury have proved amenable to HBO. One-hour-long hyperbaric oxygen treatment at 3 ATA, which was first applied at $24 \mathrm{~h}$ after SAH and repeated daily over 7 days, exerted no effect on cerebroventricular volume expansion (experimental hydrocephalus), as determined on day 21 in the endovascular perforation model of SAH [18]. Nevertheless, HBO improved neurological deficits in hydrocephalic animals 14 days after $\mathrm{SAH}$ and improved spatial learning and memory abilities between days 17 and 21 [18]. Although it may seem clear that $\mathrm{HBO}$ does not ameliorate hydrocephalus after $\mathrm{SAH}$, other treatment regimens (with the inclusion of acute treatment) and modified hyperbaric parameters need to be tested before an ultimate conclusion can be drawn.

\section{Hyperbaric oxygen for intracerebral hemorrhage}

Nontraumatic ICH accounts for about $15 \%$ of all strokes [19] and is associated with a 50\% mortality rate at 1 year [20]. Preclinical studies have compared different hyperbaric levels for oxygen treatments to reduce $\mathrm{ICH}$ brain injury. Following intracerebral injection of $200 \mu \mathrm{l}$ of blood, HBO 
treatment was used at 1.0, 1.8, 2.0, and 2.2 ATA to alleviate brain edema determined on the third and fifth days after hemorrhage in rabbits. These authors found that HBO at 2 ATA exerted an optimal effect. At 2 ATA, the excess in brain tissue water content over the sham level was reduced by $67.7 \%$ on day 3 and $79.5 \%$ on day 5 as compared to the untreated control [21]. The HBO at other pressures (1.8 ATA and 2.2 ATA) reduced brain water content by about $40 \%$ on the third and fifth days. A reduction in aquaporin 4 (AQP4) expression in the hemorrhagic brain accompanied the reduction in brain water content. Interestingly, the 2 ATA level has been adopted in several other studies of HBO's effects on ICH.

In addition, $\mathrm{HBO}$ for $\mathrm{ICH}$ is associated with a rather short therapeutic window of opportunity, although it appears to be longer (hence the better translational potential) in younger individuals. Zhou et al. determined that in adult mice with experimental ICH $(30 \mu \mathrm{l}$ of autologous blood injection), HBO (60 min at 3 ATA) initiated within $30 \mathrm{~min}$ but not $60 \mathrm{~min}$ after the ictus alleviated edema and improved neurological outcomes [22]. With this early HBO treatment, the excess of brain water content with respect to the nonhemorrhagic hemisphere was reduced by $50 \%$ on day 3 . Also at 3 days, early $\mathrm{HBO}$ reduced the excess of brain water by $53 \%$ after collagenase-induced bleeding $(0.03 \mathrm{U})$. In a neonatal model of hemorrhage to the subependymal germinal matrix, HBO (2.5 ATA for $1 \mathrm{~h}$ ) administered at $1 \mathrm{~h}$ after collagenase injection was associated with an improvement in cognitive function at 3 weeks after the hemorrhage (T-maze and Morris water maze testing) and in sensorimotor function at 4 weeks. HBO also had a reducing effect on brain atrophy, splenomegaly, and cardiac hypertrophy determined at 1 month after injury [23].

The effectiveness of early $\mathrm{HBO}$ against brain edema was also examined in a minipig model of $\mathrm{ICH}$ at high altitudes. The HBO (2.5 ATA for $1 \mathrm{~h}$ ) was administered 30 min after a $3 \mathrm{ml}$ blood injection. The intervention resulted in a $38.7 \%$ reduction in perihematomal edema $24 \mathrm{~h}$ after $\mathrm{ICH}$ [24]. Thus, the brain water reduction was more modest than the complete reduction achieved with 2 ATA and the $74.8 \%$ reduction with 2.2 ATA as shown previously by Wu et al. at $24 \mathrm{~h}$ after ICH in rabbits [21].

Of note, the research on $\mathrm{HBO}$ for $\mathrm{ICH}$ has addressed possible interactions between oxygen and blood degradation products, which may pose a risk of aggravating brain injury. Researchers at the University of Michigan found diverse effects of treatment with $\mathrm{HBO}$ for $\mathrm{ICH}$ (attenuated or enhanced brain edema) depending on the type of blood components administered [25]. The HBO (3.0 ATA for $1 \mathrm{~h}$ ), initiated $1 \mathrm{~h}$ after autologous blood injection, produced a beneficial effect. In contrast, HBO aggravated brain edema induced by ferrous iron (released upon clot lysis). These data may suggest that more research is needed regarding the safety of $\mathrm{HBO}$ use upon hematoma degradation and absorption phases.

\section{Hyperbaric oxygen conditioning for intracerebral hemorrhage}

One way to target $\mathrm{ICH}$-induced brain injury is $\mathrm{HBO}$ preconditioning (HBO-PC), which has also been analyzed for effectiveness. Conditioning for $\mathrm{ICH}$ allows interactions of $\mathrm{HBO}$ with the blood clot to be avoided, although currently the applicability of preconditioning is limited to a few hospital and pre-hospital scenarios. Qin et al. addressed the issue of an optimal HBO-PC regimen by using an autologous blood $(100 \mu \mathrm{l})$ model of ICH. They showed that with five 1 -h sessions of 3 ATA HBO$P C$, the increment in perihematomal brain water content was reduced by $52.1 \%$ (from $3.2 \%$ excess to $1.5 \%$ ) on day 1 , followed by a $23.2 \%$ reduction (from $4.3 \%$ to $3.3 \%$ ) on day 3 after blood injection. Two or three $\mathrm{HBO}$ sessions were ineffective [26].

In another study in rats, HBO-PC (5 sessions; $5 \mathrm{HBO}$ ) at 2 ATA reduced brain edema by $40 \%$ and $22 \%$ in the ipsilateral cortex and basal ganglia, respectively, $72 \mathrm{~h}$ after autologous blood injection $(80 \mu \mathrm{l})$. In addition, the HBO-preconditioned group exhibited a significantly reduced neuronal degeneration count around the hematoma, decreased by $20.7,13.3$, and $14.6 \%$ as compared to the $\mathrm{ICH}$ group at $12 \mathrm{~h}, 24 \mathrm{~h}$, and $24 \mathrm{~h}$, respectively [27].

The $\mathrm{ICH}$ produced with a smaller hematoma volume $(50 \mu \mathrm{l})$ in rats was preconditioned with $5 \mathrm{HBO}$ at 2 ATA. The conditioning reduced excessive peri-hematoma brain water content by $53.9 \%$ at $72 \mathrm{~h}$ after the hemorrhage [28] as compared to the untreated group.

In these studies, preconditioning yielded smaller reductions in brain water content than with early post-treatment with $\mathrm{HBO}$ at 2 ATA, associated with a $67.7 \%$ reduction rate on day 3 [21]. Likewise, the reductions in AQP4 expression were smaller with 2 ATA HBO-PC as compared to 2 ATA HBO treatment: $39.2 \%$ vs. $66.7 \%$ and $52.83 \%$ vs. $89 \%$, as determined on days 3 and 5 , respectively $[21,28]$.

These results may indicate greater efficacy of $\mathrm{HBO}$ when it is given after the hemorrhage as compared to its use as preconditioning. The findings may also indicate synergy of HBO post-treatment mechanisms with endogenous defenses that are activated by the injury itself.

In addition, the postconditioning approach with $\mathrm{HBO}$ should be considered after the hemorrhage in order to stimulate brain adaptation to injurious sequelae of the bleeding [29-31]. The HBO post- 
conditioning stimulus would most likely be the repeated HBO exposures, of short duration, and ATA levels that are relatively high although adjusted so as not to induce convulsions $[32,33]$. Such a procedure applied shortly after the bleeding could rapidly activate antioxidant and anti-inflammatory defenses, later used against blood-induced oxidative stress and neuroinflammation.

It can be quite insightful to compare the antiedematous effects of $\mathrm{HBO}$ in cerebral hemorrhage and cerebral ischemia. When HBO at 2.5 ATA for $2 \mathrm{~h}$ was initiated at $1 \mathrm{~h}$ after endothelin-induced focal cerebral ischemia, a $38.6 \%$ reduction in excess brain water content was noted in the ischemic hemisphere at $24 \mathrm{~h}$ [34]. One hour of 3 ATA HBO administered after $2 \mathrm{~h}$ middle cerebral artery occlusion (MCAO) in rats led to a $43.1 \%$ reduction in focal edema volume at $24 \mathrm{~h} \mathrm{[35].} \mathrm{This} 30-40 \%$ edema reduction range with $\mathrm{HBOT}$ (or closer to $20 \%$ in permanent ischemia) has been reported by most experimental studies of focal ischemic stroke $[36,37]$. Thus, reductions in brain water content with $\mathrm{HBO}$ seem smaller after focal brain ischemia than after hemorrhage, with over $70 \%$ reductions reported with $\mathrm{HBOT}$ and $50 \%$ with HBO-PC at $24 \mathrm{~h}$ after hemorrhage $[21,26]$. This result may indicate that relatively mild, predominantly perihematomal edema after hemorrhage more effectively responds to HBOT.

\section{Clinical findings}

Clinical treatment for brain edema after ICH remains mostly supportive (steroids, hyperventilation, mannitol). Several major clinical trials of investigational therapies for ICH did not use hyperbaric oxygen. Those studies, such as INTERACT-2, ATACH-II and CLEAR-III, yielded predominantly disappointing results. INTERACT-2 (Intensive Blood Pressure Reduction in Acute Cerebral Hemorrhage Trial 2) investigators reported that the early, intensive lowering of blood pressure (BP) did not result in reduced rates of the primary outcomes [38]. Also the ATACH-II (Antihypertensive Treatment of Acute Cerebral Hemorrhage II) trial showed no benefits of intensive BP lowering in patients with intracranial hemorrhage [39]. CLEAR III (Clot Lysis: Evaluating Accelerated Resolution of Intraventricular Hemorrhage Phase III) tested the use of alteplase to quickly remove blood from ventricles after ICH, which however did not significantly improve the primary endpoints [40]. In STICH (Surgical Trial in Intracerebral Haemorrhage) no overall improved clinical benefit was seen from early hematoma evacuation [41].

Limited benefits from clinical trials conducted so far for brain hemorrhage leave room for testing other therapies, and HBO is certainly one of the treatments to consider. Previous authors have suggested that responsiveness to HBO may help to determine the surgical intervention for putaminal hemorrhage [42]. Others have suggested a role of $\mathrm{HBO}$ in arresting the spread of hemorrhage [43]. Moreover, more recent clinical trials examined the effect of HBO on ischemic and hemorrhagic stroke. Repeated sessions of hyperbaric oxygen (40 daily sessions, 5 days/week, 90 min each, 100\% oxygen at 2 ATA), initiated 6-36 months after stroke, improved neurological functions and life quality of patients with hemorrhagic or ischemic stroke [44]. Multiple HBO treatments (40-60x at 2 ATA) started 3-180 months (median: 19-23 months) after stroke also reduced memory impairments in hemorrhagic and ischemic strokes [45].

However, $\mathrm{HBO}$ also shows repair and regeneration potential in clinical studies of neurologic conditions other than brain hemorrhages, including autism, traumatic brain injury and post-concussive symptoms (PCS). All these studies stress the repair and regeneration properties of HBOT. Also, hyperbaric oxygen therapy has long been cleared by the FDA and Undersea and Hyperbaric Medical Society for use in decompression sickness (DCS) by countering pathophysiological sequelae of decompression including injury to the endothelia [46]. Studies have shown that HBO may regenerate endothelial function, which may provide an important mechanism of HBOT for DCS [47].

There are several positive studies of $\mathrm{HBO}$ for PCS. A study by Boussi-Gross of HBO for PCS after mild traumatic brain injury showed an improvement with a delayed treatment of 40 to 60 daily HBO sessions at 2 ATA [48]. However, the study titled "Hyperbaric Oxygen Therapy (HBO2T) for Post-Concussive Symptoms (PSC) After Mild Traumatic Brain Injury (mTBI)" used 40 once-daily hyperbaric oxygen sessions at 2 ATA over a 10-week period and found no effect on post-concussive symptoms, whereas an improved total score in the hyperbaric groups could be due to nonspecific effects [49]. As for those ineffective trials, inappropriate sham groups and small sample size have been a concern to other researchers [50]. Lack of effectiveness of HBO for PCS in some studies was suggested to depend of several factors including a delayed treatment time [50]. Likewise, even though the "Hyperbaric Oxygen Therapy in Chronic Stable Brain Injury" (HYBOBI) study reported safety of HBOT (60 daily HBO sessions at 1.5 ATA $100 \%$ oxygen) for stroke, anoxia or trauma, the neurological results were inconclusive, despite many participants showing improvement. The investigators expressed a need for more compelling evidence from carefully designed clinical trials [51].

In one study of HBO for autistic children it was noted that the participants improved on the Clinical Global Impression scale as well as the Autism Treatment Evaluation Checklist (ATEC) with 40 hyperbaric treatments at $1.3 \mathrm{~atm}$ [52]. Another clin- 
ical trial confirmed improved cerebral flow in the white matter of children and improved neurological performance on ATEC [53]. In addition, by now there have been around 10 positive studies of HBOT for cerebral palsy, with over 600 participants [54]. However, it needs to be noted that 40 HBO treatments at 1.5 ATA over an 8-week period in children with cerebral palsy was no more effective than hyperbaric air (HBA) in improving motor function [55]. Others have pointed out an improvement in both HBA and $\mathrm{HBO}$ groups in that study, and argued that $14 \%$ oxygen at 1.5 ATA should not be considered a placebo group, while the role of hyperbaria alone should be meticulously addressed in further studies [54, 56].

It is difficult not to note that novel clot removal approaches may create new opportunities for HBO use for these patients. With the clot gone, the risk of interactions between oxygen and blood degradation products is much attenuated. New research will evaluate whether oxygen side effects can indeed be reduced after blood clot evacuation. Genetic factors which play a role in cerebral hemorrhages [57] should also be considered as potentially determining the response to HBOT.

As for SAH, studies have shown that clinical outcomes of endovascular coiling of a ruptured aneurysm may even surpass those associated with open skull surgery [58]. The advent of endovascular coiling also creates a more suitable therapeutic option than surgery for a combination with $\mathrm{HBO}$ to improve outcomes, which should be further tested clinically.

\section{Future research directions}

To evaluate HBO-based modalities and optimal treatment regimens for hemorrhagic stroke, experimental studies should calculate percent reductions in brain water contents as well as other indexes of brain protection with the treatment.

Further research will establish the hemorrhagic injury characteristics that are most responsive to HBO treatment. The use of MRI, where available, may increase quantitation confidence, by allowing total lesion assessment between treated and untreated groups, and also stratifying the impact of $\mathrm{HBO}$ depending on revealed penumbral patterns. New translational research needs to evaluate the effectiveness of HBO for hemorrhage with comorbidities, which may challenge the results most often established in young and healthy laboratory animals [59].

In addition, studying the effect of HBO on hemorrhage-induced brain inflammation appears to be a plausible research direction. The inflammatory response greatly contributes to perihematomal edema and secondary brain injury after $\mathrm{ICH}[60]$. The HBO, by reducing HIF- $1 \alpha$ expression and func- tion, may target regulatory mechanisms of inflammatory cells dependent on HIF-1 [61]. Translational studies showed that $\mathrm{HBO}$ effectively reduced inflammation in the brain in traumatic brain injury and cerebral ischemia as well [62-64]. As for underlying mechanisms, $\mathrm{HBO}$ reduces excessive microglial activation [65], reduces proinflammatory cytokines IL-1 $\beta$ and TNF- $\alpha$, and activates anti-inflammatory IL-10 $[62,66]$.

One clinical study showed markers of reduced inflammation in patients pretreated with $\mathrm{HBO}$ at 2.4 ATA before cardiac procedures on cardiopulmonary bypass [67], and several clinical trials directed at targeting diseases of musculoskeletal and gastrointestinal systems with an inflammatory component also showed clinical improvement of treated patients $[68,69]$. The potential of HBO to target inflammation, and thereby to prevent the exacerbation of hemorrhagic injuries, needs to be further verified.

The effects of $\mathrm{HBO}$ on hematoma enlargement additionally need to be examined in further studies. To this end, new models replicating these phenomena are needed [70, 71]. Another issue to be determined is what mechanisms of $\mathrm{HBO}$-induced tissue regeneration are at play after hemorrhage [4].

Regardless of the options pursued, good laboratory practice needs to be maintained for all preclinical studies to advance to clinical trials. To this end, the updated STAIR and RIGOR criteria provide guidelines that are useful in preclinical hemorrhagic stroke research, despite being originally focused on ischemic stroke [72].

\section{Conclusions}

Preclinical research shows that HBO exerts a neuroprotective effect against hemorrhagic brain injuries. The impact of HBO on the brain water content and other indices, as reviewed here, reveals a higher effectiveness of the post-treatment approach involving moderate pressures of oxygen ( 2 ATA) in experimental ICH, and a tendency to higher pressures for beneficial effects to occur after SAH. A short therapeutic window characterizes both applications, however.

The robust brain-protective effects of $\mathrm{HBO}$ for hemorrhagic injuries, not limited to relief from hypoxia, may justify its use for hemorrhagic stroke, which awaits verification in clinical trials.

\section{Conflict of interest}

The authors declare no conflict of interest.

\section{References}

1. Ostrowski RP, Stepien K, Pucko E, Matyja E. Hyperbaric oxygen modalities are differentially effective in distinct brain ischemia models. Med Gas Res 2016; 6: 39-47. 
2. Jiang Y, Wu J, Keep RF, Hua Y, Hoff JT, Xi G. Hypoxia-inducible factor-1alpha accumulation in the brain after experimental intracerebral hemorrhage. J Cereb Blood Flow Metab 2002; 22: 689-96.

3. Thiex R, Tsirka SE. Brain edema after intracerebral hemorrhage: mechanisms, treatment options, management strategies, and operative indications. Neurosurg Focus 2007; 22: E6.

4. Peng ZR, Yang AL, Yang QD. The effect of hyperbaric oxygen on intracephalic angiogenesis in rats with intracerebral hemorrhage. J Neurol Sci 2014; 342: 114-23.

5. Krishnamurthi RV, Moran AE, Forouzanfar MH, et al. The global burden of hemorrhagic stroke: a summary of findings from the GBD 2010 study. Glob Heart 2014; 9 : 101-6.

6. MacDonald E. Aneurysmal subarachnoid hemorrhage. J Neurosci Nurs 1989; 21: 313-21.

7. Fewel ME, Thompson BG Jr, Hoff JT. Spontaneous intracerebral hemorrhage: a review. Neurosurg Focus 2003; 15: E1.

8. Zhang YP, Cai J, Shields LB, Liu N, Xu XM, Shields CB. Traumatic brain injury using mouse models. Transl Stroke Res 2014; 5: 454-71.

9. Krafft PR, McBride DW, Lekic T, et al. Correlation between subacute sensorimotor deficits and brain edema in two mouse models of intracerebral hemorrhage. Behav Brain Res 2014; 264: 151-60.

10. Wiebers DO, Feigin VL, Brown J RD. Handbook of Stroke. PA Lippincott Williams \& Wilkins Philadelphia 2006.

11. van Gijn J, Rinkel GJ. Subarachnoid haemorrhage: diagnosis, causes and management. Brain 2001; 124: 249-78.

12. Jarus-Dziedzic K, Czernicki Z, Kozniewska E. Acute decrease of cerebrocortical microflow and lack of carbon dioxide reactivity following subarachnoid haemorrhage in the rat. Acta Neurochir Suppl 2003; 86: 473-6.

13. Kohshi K, Yokota A, Konda N, Munaka M, Yasukouchi H. Hyperbaric oxygen therapy adjunctive to mild hypertensive hypervolemia for symptomatic vasospasm. Neurol Med Chir (Tokyo) 1993; 33: 92-9.

14. Hu Q, Wu C, Chen JY, Yan F, Li JR, Chen G. The relationship between hypoxia-inducible factor-1alpha expression and apoptosis in early brain injury after subarachnoid hemorrhage. Zhejiang Da Xue Xue Bao Yi Xue Ban 2014; 43: 58-65.

15. Ostrowski RP, Colohan AR, Zhang JH. Mechanisms of hyperbaric oxygen-induced neuroprotection in a rat model of subarachnoid hemorrhage. J Cereb Blood Flow Metab 2005; 25: 554-71.

16. Ostrowski RP, Tang J, Zhang JH. Hyperbaric oxygen suppresses NADPH oxidase in a rat subarachnoid hemorrhage model. Stroke 2006; 37: 1314-8.

17. Kocaogullar Y, Ustun ME, Avci E, Karabacakoglu A, Fossett $D$. The role of hyperbaric oxygen in the management of subarachnoid hemorrhage. Intensive Care Med 2004; 30: 141-6.

18. Hu Q, Vakhmjanin A, Li B, Tang J, Zhang JH. Hyperbaric oxygen therapy fails to reduce hydrocephalus formation following subarachnoid hemorrhage in rats. Med Gas Res 2014; 4: 12.

19. Soltani A, Clark WM, Hansmann DR. Sonothrombolysis: an emerging modality for the treatment of acute ischemic and hemorrhagic stroke. Transl Stroke Res 2011; 2: 159-170.

20. Fiorella D, Zuckerman SL, Khan IS, Ganesh NK, Mocco J. Intracerebral hemorrhage: a common and devastating disease in need of better treatment. World Neurosurg 2015; 84: 1136-41.
21. Wu J, Chen J, Guo H, Peng F. Effects of high-pressure oxygen therapy on brain tissue water content and AQP4 expression in rabbits with cerebral hemorrhage. Cell Biochem Biophys 2014; 70: 1579-84.

22. Zhou W, Marinescu M, Veltkamp R. Only very early oxygen therapy attenuates posthemorrhagic edema formation and blood-brain barrier disruption in murine intracerebral hemorrhage. Neurocrit Care 2015; 22: 121-32.

23. Lekic T, Manaenko A, Rolland W, et al. Beneficial effect of hyperbaric oxygenation after neonatal germinal matrix hemorrhage. Acta Neurochir Suppl 2011; 111: 253-7.

24. Zhu HT, Bian C, Yuan JC, et al. Hyperbaric oxygen therapy ameliorates acute brain injury after porcine intracerebral hemorrhage at high altitude. Crit Care 2015; 19: 255.

25. Qin Z, Xi G, Keep RF, Silbergleit R, He Y, Hua Y. Hyperbaric oxygen for experimental intracerebral hemorrhage. Acta Neurochir Suppl 2008; 105: 113-7.

26. Qin Z, Song S, Xi G, et al. Preconditioning with hyperbaric oxygen attenuates brain edema after experimental intracerebral hemorrhage. Neurosurg Focus 2007; 22: E13.

27. Yang L, Tang J, Chen Q, et al. Hyperbaric oxygen preconditioning attenuates neuroinflammation after intracerebral hemorrhage in rats by regulating microglia characteristics. Brain Res 2015; 1627: 21-30.

28. Fang J, Li H, Li G, Wang L. Effect of hyperbaric oxygen preconditioning on peri-hemorrhagic focal edema and aquaporin-4 expression. Exp Ther Med 2015; 10: 699-704.

29. Sandu N, Schaller B. Postconditioning: a new or old option after ischemic stroke? Expert Rev Cardiovasc Ther 2010; 8: 479-82.

30. Schaller B, Graf R, Jacobs AH. Ischaemic tolerance: a window to endogenous neuroprotection? Lancet 2003; 362: 1007-8.

31. Wang J, Han D, Sun M, Feng J. A Combination of remote ischemic perconditioning and cerebral ischemic postconditioning inhibits autophagy to attenuate plasma HMGB1 and induce neuroprotection against stroke in rat. J Mol Neurosci 2016; 58: 424-31.

32. Karlinski M, Gluszkiewicz M, Czlonkowska A. The accuracy of prehospital diagnosis of acute cerebrovascular accidents: an observational study. Arch Med Sci 2015; 11: $530-5$

33. Liu W, Li J, Sun X, et al. Repetitive hyperbaric oxygen exposures enhance sensitivity to convulsion by upregulation of eNOS and nNOS. Brain Res 2008; 1201: 128-34.

34. Huang ZX, Kang ZM, Gu GJ, et al. Therapeutic effects of hyperbaric oxygen in a rat model of endothelin-1-induced focal cerebral ischemia. Brain Res 2007; 1153: 204-13.

35. Veltkamp R, Siebing DA, Sun L, et al. Hyperbaric oxygen reduces blood-brain barrier damage and edema after transient focal cerebral ischemia. Stroke 2005; 36 : 1679-83.

36. Liu JR, Zhao Y, Patzer A, et al. The c-Jun N-terminal kinase (JNK) inhibitor XG-102 enhances the neuroprotection of hyperbaric oxygen after cerebral ischaemia in adult rats. Neuropathol Appl Neurobiol 2010; 36: 211-24.

37. Sunami K, Takeda Y, Hashimoto M, Hirakawa M. Hyperbaric oxygen reduces infarct volume in rats by increasing oxygen supply to the ischemic periphery. Crit Care Med 2000; 28: 2831-6.

38. Anderson CS, Heeley E, Huang Y, et al. Rapid blood-pressure lowering in patients with acute intracerebral hemorrhage. N Engl J Med 2013; 368: 2355-65.

39. Qureshi Al, Palesch YY, Martin R, et al. Interpretation and Implementation of Intensive Blood Pressure Reduc- 
tion in Acute Cerebral Hemorrhage Trial (INTERACT II). J Vasc Interv Neurol 2014; 7: 34-40.

40. Hanley D. Clot Lysis: Evaluating Accelerated Resolution of Intraventricular Hemorrhage (CLEAR III) Results. In: International Stroke Conference 2016; 02-18; Los Angeles. 2016.

41. Potts MB, Riina HA. Refining the role for evacuation of spontaneous intracerebral hematomas: results of STICH II. World Neurosurg 2014; 82: 549-50.

42. Kanno T, Nonomura K. Hyperbaric oxygen therapy to determine the surgical indication of moderate hypertensive intracerebral hemorrhage. Minim Invasive Neurosurg 1996; 39: 56-9.

43. Al-Waili NS, Butler GJ, Beale J, et al. Hyperbaric oxygen in the treatment of patients with cerebral stroke, brain trauma, and neurologic disease. Adv Ther 2005; 22 : 659-78.

44. Efrati S, Fishlev G, Bechor Y, et al. Hyperbaric oxygen induces late neuroplasticity in post stroke patients: randomized, prospective trial. PLoS One 2013; 8: e53716.

45. Boussi-Gross R, Golan H, Volkov O, et al. Improvement of memory impairments in poststroke patients by hyperbaric oxygen therapy. Neuropsychology 2015; 29: 610-21.

46. Brubakk AO, Ross JA, Thom SR. Saturation diving; physiology and pathophysiology. Compr Physiol 2014; 4: 1229-72.

47. Thom SR: Hyperbaric oxygen: its mechanisms and efficacy. Plast Reconstr Surg 2011; 127 Suppl 1: 131S-141S.

48. Boussi-Gross R, Golan H, Fishlev G, et al. Hyperbaric oxygen therapy can improve post concussion syndrome years after mild traumatic brain injury - randomized prospective trial. PLoS One 2013; 8: e79995.

49. Cifu DX, Hart BB, West SL, Walker W, Carne W. The effect of hyperbaric oxygen on persistent postconcussion symptoms. J Head Trauma Rehabil 2014; 29: 11-20.

50. Hu Q, Manaenko A, Guo Z, Huang L, Tang J, Zhang JH. Hyperbaric oxygen therapy for post concussion symptoms: issues may affect the results. Med Gas Res 2015; 5: 10.

51. Churchill S, Weaver LK, Deru K, et al. A prospective trial of hyperbaric oxygen for chronic sequelae after brain injury (HYBOBI). Undersea Hyperb Med 2013; 40: 165-93.

52. Rossignol DA, Rossignol LW, Smith S, et al. Hyperbaric treatment for children with autism: a multicenter, randomized, double-blind, controlled trial. BMC Pediatr 2009; 9: 21.

53. El-baz F, Elhossiny RM, Azeem YA, Girgis M. Study the effect of hyperbaric oxygen therapy in Egyptian autistic children: a clinical trial. Egypt J Med Human Genet 2014; 15: 155-62.

54. Marois P. Hyperbaric oxygen treatment. Ann Neurol 2013; 74: 149.

55. Lacey DJ, Stolfi A, Pilati LE. Effects of hyperbaric oxygen on motor function in children with cerebral palsy. Ann Neurol 2012; 72: 695-703.

56. Mychaskiw $G 2^{\text {nd }}$. How many deaths will it take till they know? Monkeys, madmen and the standard of evidence. Undersea Hyperb Med 2012; 39: 795-7.

57. Szpecht D, Szymankiewicz M, Seremak-Mrozikiewicz A, Gadzinowski J. The role of genetic factors in the pathogenesis of neonatal intraventricular hemorrhage. Folia Neuropathol 2015; 53: 1-7.

58. Kozba-Gosztyla M, Czapiga B, Jarmundowicz W. Aneurismal subarachnoid hemorrhage: who remains for surgical treatment in the post-ISAT era? Arch Med Sci 2015; 11: 536-43.

59. Snarska KK, Bachórzewska-Gajewska H, Kapica-Topczewska K, et al. Hyperglycemia and diabetes have dif- ferent impacts on outcome of ischemic and hemorrhagic stroke. Arch Med Sci 2017; 13: 100-8.

60. Rolland WB, Lekic T, Krafft PR, et al. Fingolimod reduces cerebral lymphocyte infiltration in experimental models of rodent intracerebral hemorrhage. Exp Neurol 2013; 241: 45-55.

61. Cramer T, Yamanishi Y, Clausen BE, et al. HIF-1alpha is essential for myeloid cell-mediated inflammation. Cell 2003; 112: 645-57.

62. Chen LF, Tian YF, Lin CH, Huang LY, Niu KC, Lin MT. Repetitive hyperbaric oxygen therapy provides better effects on brain inflammation and oxidative damage in rats with focal cerebral ischemia. J Formos Med Assoc 2014; 113: 620-8.

63. Lee YS, Chio CC, Chang CP, et al. Long course hyperbaric oxygen stimulates neurogenesis and attenuates inflammation after ischemic stroke. Mediators Inflamm 2013; 2013: 512978.

64. Ostrowski RP, Pucko EB. Research of medical gases in Poland. Med Gas Res 2013; 3: 17

65. Lim SW, Wang CC, Wang YH, Chio CC, Niu KC, Kuo JR. Microglial activation induced by traumatic brain injury is suppressed by postinjury treatment with hyperbaric oxygen therapy. J Surg Res 2013; 184: 1076-84.

66. Chen X, Duan XS, Xu LJ, et al. Interleukin-10 mediates the neuroprotection of hyperbaric oxygen therapy against traumatic brain injury in mice. Neuroscience 2014; 266: 235-43.

67. Alex J, Laden G, Cale AR, et al. Pretreatment with hyperbaric oxygen and its effect on neuropsychometric dysfunction and systemic inflammatory response after cardiopulmonary bypass: a prospective randomized double-blind trial. J Thorac Cardiovasc Surg 2005; 130: 1623-30.

68. Efrati S, Golan H, Bechor Y, et al. Hyperbaric oxygen therapy can diminish fibromyalgia syndrome: prospective clinical trial. PLoS One 2015; 10: e0127012.

69. Rossignol DA. Hyperbaric oxygen treatment for inflammatory bowel disease: a systematic review and analysis. Med Gas Res 2012; 2: 6.

70. MacLellan CL, Silasi G, Auriat AM, Colbourne F. Rodent models of intracerebral hemorrhage. Stroke 2010; 41: S95-98.

71. Mracsko E, Veltkamp R. Neuroinflammation after intracerebral hemorrhage. Front Cell Neurosci 2014; 8: 388.

72. Kellner CP, Connolly ES Jr. Neuroprotective strategies for intracerebral hemorrhage: trials and translation. Stroke 2010; 41: S99-102. 